\title{
A solution to the hierarchy problem from an almost decoupled hidden sector within a classically scale invariant theory
}

\author{
Robert Foot,,$* *$ Archil Kobakhidze, ${ }^{1,+}$ Kristian L. McDonald, $2,+$ and Raymond R. Volkas $1, \S$ \\ ${ }^{1}$ School of Physics, Research Centre for High Energy Physics, \\ University of Melbourne, Victoria 3010, Australia. \\ ${ }^{2}$ Theory Group, TRIUMF, 4004 Wesbrook Mall, Vancouver, BC V6T2A3, Canada.
}

\begin{abstract}
If scale invariance is a classical symmetry then both the Planck scale and the weak scale should emerge as quantum effects. We show that this can be realized in simple scale invariant theories with a hidden sector. The weak/Planck scale hierarchy emerges in the (technically natural) limit where the hidden sector decouples from the ordinary sector. The weak scale is protected from quadratic divergences because of classical scale invariance, so there is no hierarchy problem.
\end{abstract}

\footnotetext{
*Email: rfoot@unimelb.edu.au

†Email: archilk@unimelb.edu.au

†Email: klmcd@triumf.ca

§Email: raymondv@unimelb.edu.au
} 


\section{INTRODUCTION}

One way to stabilize the electroweak scale against radiative corrections is to promote scale invariance to a good classical symmetry by eliminating the $\mu^{2}$ mass parameter from the Higgs potential [1]. As first discussed by Coleman and E. Weinberg [2], electroweak symmetry breaking can still arise since scale invariance is anomalous and the radiatively corrected Higgs potential may induce spontaneous symmetry breaking. When applied to the minimal standard model this elegant idea fails as the heavy top quark precludes the required dominance of the bosonic contributions to the effective potential.

Additional bosonic contributions, in the form of extra gauge bosons and/or scalars, can lead to phenomenologically successful models $[3,4,4,5,6,7,8]$ of electroweak symmetry breaking - with the electroweak scale stabilized against radiative corrections. A pressing issue though, is what to do about gravity, which of course, also involves a scale - the Planck mass. One possibility is that gravity can be treated separately from the other interactions, and we need not worry that the gravitational interaction explicitly violates scale invariance. Alternatively, it is tempting to apply scale invariance to the the whole fundamental theory - including gravity.

We propose that the Planck mass arises from the vacuum expectation value (VEV) of a scalar field $S$ [9], which is dynamically generated via the radiative breaking of scale invariance. ${ }^{1}$ Thus we include gravity by postulating the scale-invariant interaction term

$$
\mathcal{L}=\sqrt{-g}\left[\frac{1}{2} \xi S^{2} R\right] .
$$

Standard gravity emerges if $\xi\langle S\rangle^{2} \equiv M_{P l}^{2}$, which requires $\langle S\rangle \sim M_{P l}$ for $\xi \sim 1$ [here $M_{P l} \equiv$ $(8 \pi G)^{-1 / 2}=2.436 \times 10^{18} \mathrm{GeV}$ is the reduced Planck Mass]. Due to its large VEV, $S$ should not couple directly to the standard particles, so we require it to be an $S U(3)_{c} \otimes S U(2)_{L} \otimes U(1)_{Y}$ gauge singlet.

Observe that the scalar $S$ only couples to the ordinary particles through the Higgs quartic coupling, ${ }^{2}$

$$
\lambda_{X} \phi^{\dagger} \phi S^{2}
$$

where $\phi$ is the standard model Higgs doublet. In the limit $\lambda_{X} \rightarrow 0$ the scalar potential separates:

$$
V(\phi, S)=V(\phi)+V(S)
$$

In this limit, $V(\phi)$ is simply the Coleman-Weinberg potential, and given the heavy top quark, spontaneous symmetry breaking in $V(\phi)$ does not arise. Thus, we end up with a massless Higgs

\footnotetext{
${ }^{1}$ Note that demanding classical scale invariance motivates the absence of the usual Einstein-Hilbert Lagrangian $\mathcal{L} \propto \sqrt{-g} M_{P l}^{2} R$, which must otherwise be arbitrarily omitted in models which spontaneously generate the Planck scale [9].

${ }^{2}$ There are also indirect couplings which are due to kinetic mixing of $S^{\prime} \equiv S-\langle S\rangle$ with the trace part of the graviton field, as we discuss later.
} 
particle. The $V(S)$ part can undergo spontaneous symmetry breaking (depending on the particle content in the 'hidden sector'), leading to $\langle S\rangle \neq 0$, and this VEV sets the Planck scale and gravity via Eq.(11). If $\lambda_{X}$ is small, but non-zero, then the symmetry breaking can be communicated to the electroweak sector. In fact it is also possible to have the $\lambda_{X}$ term induce symmetry breaking in both $\phi$ and $S$. Either way, the ratio of scales $\langle\phi\rangle /\langle S\rangle$ is controlled by just the one adjustable parameter $\lambda_{X}$. Furthermore, the physically interesting limit, where $\langle\phi\rangle /\langle S\rangle \sim M_{W} / M_{P l} \rightarrow 0$, corresponds to the technically natural ${ }^{3}$ limit of $\lambda_{X} \rightarrow 0$. This would give a technically natural solution to the hierarchy problem.

The outline of this paper is as follows: In Sec. \we examine the radiatively corrected Higgs potential of the simplest models which have the weak and Planck scales arising from the anomalous breaking of scale invariance. As will be discussed there, such models feature a pseudo-Goldstone boson (PGB) which is associated with the breaking of scale invariance. This PGB is the main observable new physics predicted by the model. In Sec. [III we investigate the coupling of this resultant pseudo-Goldstone boson to ordinary matter and discuss the constraints from experiments. We comment on the cosmological constant, which vanishes classically within our model, in Sec.IV and conclude in Sec. $\mathrm{V}$.

\section{HIGGS POTENTIAL ANALYSIS}

We first consider the simplest possibility of the addition of one real scalar, $S$, which couples to the curvature as in Eq. (1). Some of this discussion is very similar to that given in Ref. [7].

The tree level scalar potential is

$$
V=\frac{\lambda_{\phi}}{2}\left(\phi^{\dagger} \phi\right)^{2}+\frac{\lambda_{S}}{8} S^{4}-\frac{\lambda_{X}}{2}\left(\phi^{\dagger} \phi\right) S^{2},
$$

and we parameterize the fields in unitary gauge as

$$
\phi=\frac{r}{\sqrt{2}}\left(\begin{array}{c}
0 \\
\sin \omega
\end{array}\right), \quad S=r \cos \omega .
$$

In this parameterization, the potential of Eq. (4) is rewritten as

$$
V_{0}(r, \omega)=r^{4}\left(\frac{\lambda_{\phi}}{8} \sin ^{4} \omega+\frac{\lambda_{S}}{8} \cos ^{4} \omega-\frac{\lambda_{X}}{4} \sin ^{2} \omega \cos ^{2} \omega\right) .
$$

\footnotetext{
${ }^{3}$ In the absence of gravity, decoupling a sector increases the symmetry of the theory, because the action $S=$ $\int d^{4} x \mathcal{L}_{\text {vis }}(x)+\int d^{4} x^{\prime} \mathcal{L}_{\text {hid }}\left(x^{\prime}\right)$ is invariant under independent Poincare transformations for the visible and hidden sectors. In the presence of gravity, this is no longer the case. However, as discussed further below, we are treating gravity classically in this analysis, so radiative corrections due to quantum gravity are ignored. We are assuming that these quantum gravity effects are sufficiently small so as to not spoil the technical naturalness arising from switching off the non-gravitational couplings between the sectors.
} 
The radial component $r$ of the Higgs fields, Eq. (5), factors out due to the absence of the tree-level mass parameter. This classical potential receives quantal radiative corrections in the manner of Coleman and Weinberg [2]. We shall work in the parameter regime where the 1-loop-level correction is sufficiently accurate. In general, the minimization of even a 1-loop-corrected effective potential involving multiple scalars cannot be done analytically, so we instead follow the approximation scheme introduced in [10] which is suitable in weakly coupled scale-invariant theories.

Following [10], we first ignore the perturbatively small radiative corrections and minimize the tree-level potential of Eq. (6). Taking the parameter regime $\lambda_{X}>0$, letting $\langle r\rangle$ be nonzero but arbitrary, the minimum is

$$
\frac{\langle\phi\rangle^{2}}{\langle S\rangle^{2}} \equiv \frac{\left\langle\tan ^{2} \omega\right\rangle}{2}=\frac{1}{2} \frac{\lambda_{X}(\Lambda)}{\lambda_{\phi}(\Lambda)},
$$

with

$$
\lambda_{X}(\Lambda)=\sqrt{\lambda_{\phi}(\Lambda) \lambda_{S}(\Lambda)}
$$

The relation, Eq. (8), is not a fine-tuning but rather the definition of a renormalization point $\mu=\Lambda$ [10], where the running coupling constants depend on $\mu$ and $\Lambda$ is the specific value where Eq. (8) holds. Equation (8) trades a dimensionless parameter for the dimensionalful renormalisation point $\Lambda$ : the phenomenon of dimensional transmutation.

The classical potential has a flat direction described by the arbitrary $\langle r\rangle$ along the vacuum solution, which shall be removed by the radiative corrections. Since the classical potential is zero along that direction, the one-loop correction necessarily dominates there.

We shall be interested in the limit where

$$
\tan ^{2} \omega=\frac{2\langle\phi\rangle^{2}}{\langle S\rangle^{2}} \sim \frac{M_{W}^{2}}{M_{P l}^{2}} \rightarrow 0 .
$$

From Eqs. (7) we see that this limiting case occurs when $\lambda_{X} \rightarrow 0$. (The other choice $\lambda_{\phi} \rightarrow \infty$ is not phenomenologically viable.) Observe that $\lambda_{X} \rightarrow 0$ corresponds to the limit where the hidden sector completely decouples from the ordinary sector. As noted earlier, this is technically natural since all non-gravitational radiative corrections to $\lambda_{X}$ must vanish in the limit where the hidden sector is completely decoupled.

To calculate the tree-level masses, we expand the Higgs potential, Eq. (4), around the vacuum: $\phi=\langle\phi\rangle+\phi^{\prime}, S=\langle S\rangle+S^{\prime}$. Of the two physical scalars, only one gains mass at tree-level; the other is classically massless due to the flat direction. Calling the state that develops mass at treelevel $H$, and the tree-level-massless state $\sigma$ (the pseudo-Goldstone Boson (PGB) of broken scale invariance), we obtain

$$
M_{H}^{2}=\lambda_{\phi} v^{2}+\lambda_{X} v^{2}, \quad H=-\cos \omega \phi_{0}^{\prime}+\sin \omega S^{\prime},
$$

(here $v \approx 246 \mathrm{GeV}$ is the electroweak VEV) and the PGB field is $\sigma=\sin \omega \phi_{0}^{\prime}+\cos \omega S^{\prime}$. Note that in the $\lambda_{X} \rightarrow 0$ limit, the mass and interactions of $H$ reduce to the standard electroweak Higgs 
particle. (In the above we took $\lambda_{X}>0$. If we had made the opposite choice, unsuitable for our present purposes, then the usual electroweak Higgs would have been the PGB [7].)

To calculate the mass of the PGB boson $\sigma$, we add the one-loop correction to the tree-level potential of Eq. (6) along the "radial" flat-direction. It has the form [10 $]^{4}$

$$
\delta V_{1-\text { loop }}=A r^{4}+B r^{4} \log \left(\frac{r^{2}}{\Lambda^{2}}\right)
$$

where

$$
A=\frac{1}{64 \pi^{2}\langle r\rangle^{4}}\left[3 \operatorname{Tr}\left(M_{V}^{4} \ln \frac{M_{V}^{2}}{\langle r\rangle^{2}}\right)+\operatorname{Tr}\left(M_{S}^{4} \ln \frac{M_{S}^{2}}{\langle r\rangle^{2}}\right)-4 \operatorname{Tr}\left(M_{F}^{4} \ln \frac{M_{F}^{2}}{\langle r\rangle^{2}}\right)\right],
$$

and

$$
B=\frac{1}{64 \pi^{2}\langle r\rangle^{4}}\left[3 \operatorname{Tr} M_{V}^{4}+\operatorname{Tr} M_{S}^{4}-4 \operatorname{Tr} M_{F}^{4}\right] .
$$

The traces sum over all degrees of freedom, with $M_{V, S, F}$ being the tree-level masses for vectors, scalars and fermions, respectively.

The stationary condition $\left.\frac{\partial \delta V_{1-\text { loop }}}{\partial r}\right|_{r=\langle r\rangle}=0$ yields

$$
\log \left(\frac{\langle r\rangle}{\Lambda}\right)=-\frac{1}{4}-\frac{A}{2 B}
$$

Computing the PGB mass, and using Eq. (14), one finds [10]:

$$
\begin{aligned}
M_{\sigma}^{2} & =\left.\frac{\partial^{2} \delta V_{1-\text { loop }}}{\partial r^{2}}\right|_{r=\langle r\rangle}=8 B\langle r\rangle^{2} \\
& =\frac{1}{8 \pi^{2}\langle r\rangle^{2}}\left[3 \operatorname{Tr} M_{V}^{4}+\operatorname{Tr} M_{S}^{4}-4 \operatorname{Tr} M_{F}^{4}\right] .
\end{aligned}
$$

Evaluating the traces, we obtain

$$
M_{\sigma}^{2} \simeq \frac{1}{8 \pi^{2}\langle r\rangle^{2}}\left[M_{H}^{4}+6 M_{W}^{4}+3 M_{Z}^{4}-12 M_{t}^{4}\right] .
$$

The constraint $M_{\sigma}^{2}>0$, implies that $M_{H}^{4}>12 M_{t}^{4}-6 M_{W}^{4}-3 M_{Z}^{4}$, evaluated at the scale $\Lambda \sim M_{W}$. Such a large Higgs mass is in conflict with the precision electroweak data. Thus additional scalar or vector bosons are required to make the model phenomenologically viable. However this is neither

\footnotetext{
${ }^{4}$ The calculation is performed within the framework of dimensional regularization of divergent integrals with the $\overline{M S}$ substraction scheme. The use of dimensional regularization is absolutely crucial in scale-invariant theories, since unlike other regularizations, it requires counterterms that preserve the form of the bare Lagrangian. Emergence of quadratic and quartic divergences in other regularization schemes (e.g. cut-off or Pauli-Villars regularizations) must be regarded as artifacts of those regularization schemes because they require counterterms which are quadratic and quartic in the regulator scale (i.e. the form of the counterterms is different form that of the bare Lagrangian).
} 
unexpected nor unwelcome since the model as it stands does not explain neutrino masses and dark matter.

While there are many ways to complete the theory to achieve those goals, it is not the purpose of the present paper to survey all the possibilities. Instead, we shall be satisfied with an example. We could, for instance, add a complex Higgs triplet, $\Delta \sim(1,3,-2)$, to ensure phenomenologicallyviable electroweak symmetry breaking and induce non-zero Majorana neutrino masses [11]. The most general Higgs potential includes the terms

$$
\mathcal{L}=\lambda \Delta^{\dagger} \Delta S^{2}+\lambda^{\prime} \phi \Delta \phi S+\text { H.c. . }
$$

The first term induces a mass for $\Delta$ whilst the second induces a VEV. This leads to neutrino masses via the coupling

$$
\mathcal{L}=\lambda_{\nu} \bar{f}_{L} \Delta\left(f_{L}\right)^{c}+\text { H.c. }
$$

where $f_{L}$ denotes a leptonic $S U(2)_{L}$ doublet and the superscript denotes CP conjugation. If the radiative contributions from $\Delta$ dominate over the other radiative contributions in the effective potential, one expects the PGB mass to be of order

$$
\begin{aligned}
M_{\sigma} & \approx \frac{\sqrt{3 \xi}}{2 \pi} \frac{M_{\Delta}^{2}}{M_{P l}} \\
& \sim\left(\frac{M_{\Delta}}{T e V}\right)^{2} 10^{-4} \mathrm{eV}
\end{aligned}
$$

Thus, we expect the mass of the PGB to range from around $10^{-4} \mathrm{eV}$ to $\sim M_{P l}$, depending on the mass of $\Delta$. The hidden sector now decouples in the limit that $\lambda_{X}, \lambda$ and $\lambda^{\prime}$ vanish. Note that only the $\lambda^{\prime}$ term in (17) breaks lepton number so the limit $\lambda^{\prime} \rightarrow 0$, in which the VEV of $\Delta$ also becomes suppressed, is technically natural. Provided $\lambda_{X}$ and $\lambda$ are both small, the hidden sector remains weakly coupled and the weak/Planck hierarchy will be preserved. Thus one expects $\sigma$ to be light, and therefore observable, in the decoupling limit.

Besides the above important phenomenological motivation for the extension of the minimal framework of our model, there is a purely theoretical one as well. The fact that the electroweak Higgs boson must be heavy $\left(M_{H} \gtrsim(12)^{1 / 4} M_{t} \approx 327 \mathrm{GeV}\right)$ and $\lambda_{X}$ is hierarchically small, implies [see Eq. (10)] that the Higgs self-interaction coupling is strong $\lambda_{\phi} \gtrsim \sqrt{3}$ at the electroweak scale. This coupling becomes non-perturbative at energies below the Planck mass. While it is not immediately obvious whether the existence of this Landau pole for $\lambda_{\phi}$ spoils the weak/Planck scale hierarchy, it is certainly desirable to have a perturbative theory at least at energies up to the Planck mass. In many extensions of the bosonic sector of our minimal model the Landau pole can be pushed beyond the Planck scale, and, in some cases, completely removed. For example, in the case of the electroweak triplet Higgs discussed above, with $M_{\Delta}>M_{H}$, the theory can be kept perturbative at all energy scales up to the Planck scale (and perhaps beyond) provided the electroweak Higgs boson is relatively light, $M_{H} \lesssim 120 \mathrm{GeV}$ or so. 
A few clarifying remarks on the calculations in this section are in order. Obviously a completely consistent approach to the problem requires the quantization of gravity. However, since classical general relativity (or its scalar-tensor extensions) does not readily admit a sensible quantum theory, we have taken in the above the widely accepted point of view that the metric may be treated as a classical background field. Moreover, we have taken this background to be (nearly) flat, $g_{\mu \nu}(x) \approx \eta_{\mu \nu}$, and performed calculations in flat spacetime. This is justified if the inverse of the local curvature radius $a$ is much smaller than the typical mass scale of the problem, because only infrared modes $(k<1 / a)$ of fields are influenced by the curvature, while the quantum effects are dominated by the ultraviolet modes $(k \gg 1 / a)$. Under these assumptions the above analysis is justified.

Let us summarize the main point concerning the hierarchy problem [1] (see also [12]). The classical scale invariance does remove all divergences that go as powers of the regulator mass scale. Consequently, no large (quadratic) corrections to the electroweak mass scale are expected and this explains its smallness in a technically-natural way (we have no fundamental explanation for the smallness of certain couplings though). In this regard, scale invariance plays the same role in the solution of the hierarchy problem as the more popular softly broken supersymmetry. Extensions of the scalar sector, which are anyway necessary to incorporate a fully realistic phenomenology (e.g. neutrino masses, dark matter, etc.), as briefly discussed above, cannot reintroduce the hierarchy problem. In these extensions, Landau poles associated with some asymptotically non-free coupling constants can be pushed beyond the Planck scale, so that the standard quantum field theory, in the domain of its applicability, is in the perturbative regime. Thus, if some fundamental theory, incorporating also a quantum theory of gravitation, possesses scale invariance in its low-energy domain (where the quantum gravitational effects decouple), the hierarchy problem can be eliminated in the way we have discussed in this section.

\section{INTERACTIONS OF THE PGB WITH ORDINARY MATTER}

The new scalar $\sigma$ which couples non-trivially with the curvature $R$ is the main new physics predicted in these models. This new physics will manifest itself though modifications to gravity at scales

$$
d \lesssim \frac{1}{M_{\sigma}}
$$

If $\sigma$ is light, $M_{\sigma} \lesssim \mathrm{eV}$, then this effect can be experimentally probed in tests of Newton's inverse square law at short distance scales. To determine the magnitude of this effect, we need to compute the coupling of the PGB scalar $\sigma$ with ordinary matter.

Consider the field $S^{\prime}$, representing the fluctuations around the vacuum. In the weak gravity limit, $R \rightarrow 0$, the gravitational field equations can be linearized, leading to [13]

$$
\frac{M_{P l}^{2}}{2}\left[-\square h_{\mu \nu}+\ldots\right]+\frac{2 M_{P l}^{2}}{\langle S\rangle}\left(\eta_{\mu \nu} \square-\nabla_{\mu} \nabla_{\nu}\right) S^{\prime}=T_{\mu \nu}^{m},
$$


where $T_{\mu \nu}^{m}$ is the stress-energy due to matter. Thus $S^{\prime}$ and the trace of $h_{\mu \nu}$ are kinetically mixed. The kinetic term can be diagonalized by introducing the field

$$
\tilde{h}_{\mu \nu} \equiv h_{\mu \nu}-\frac{1}{2} \eta_{\mu \nu} h-2 \eta_{\mu \nu} \frac{S^{\prime}}{\langle S\rangle} .
$$

This transformation to the kinetically diagonal basis, $\left(\tilde{h}, S^{\prime}\right)$, introduces a host of exotic Planck scale derivative couplings. In the Higgs potential analysis of the preceding section we have ignored this kinetic mixing, essentially doing perturbation theory in the kinetically mixed basis $\left(h_{\mu \nu}, S^{\prime}\right)$. Since we are treating gravity as a classical background field, such a procedure is reasonable. However, to determine the couplings of $\sigma=\sin \omega \phi_{0}^{\prime}+\cos \omega S^{\prime}$ to matter it is necessary to transform to the kinetically diagonal basis. In the kinetically diagonalized basis $\left(\tilde{h}, S^{\prime}\right)$, the kinetic term for for the $S^{\prime}$ field has the form

$$
\frac{1}{2}(1+6 \xi) \partial_{\mu} S^{\prime} \partial_{\mu} S^{\prime}
$$

We are primarily interested in the leading order interactions of the PGB $\sigma$ with light matter fermions, which are linear in $\sigma$. Since the kinetic terms of the fermions (as well as vector bosons) are conformally invariant, these interactions arise at the classical level through the fermion mass terms,

$$
\begin{array}{r}
\sqrt{-g} m_{f} \bar{\psi}_{f} \psi_{f} \rightarrow\left(1+\frac{1}{2} h-\frac{4 \sigma}{\langle r\rangle}\right)\left(1+\frac{3 \sigma}{\langle r\rangle}\right) m_{f} \bar{\psi}_{f} \psi_{f}= \\
m_{f} \bar{\psi}_{f} \psi_{f}-\frac{m_{f}}{\langle r\rangle} \sigma \bar{\psi}_{f} \psi_{f}
\end{array}
$$

However, the $\sigma$ interaction term is exactly cancelled by the Yukawa interactions induced by the Higgs $(\phi)-S$ mass mixing, $\frac{y_{f}}{\sqrt{2}} \sigma \sin \omega \bar{\psi}_{f} \psi_{f}=+\frac{m_{f}}{\langle r\rangle} \sigma \bar{\psi}_{f} \psi_{f}$. This remarkable cancellation is a manifestation of the underlying scale invariance and ensures that fundamental fermions are only weakly coupled to the PGB.

The non-trivial admixture of gluons inside nucleons requires us to consider $\sigma$-gluon interactions induced at the quantum level in order to examine the PGB coupling to matter. As the Weyl rescaling is anomalous we must include the anomalous trace of the energy-momentum tensor in the effective Lagrangian. Thus

$$
\sqrt{-g} T^{\mathrm{anom}} \rightarrow-4 \frac{\sigma}{\langle r\rangle}\left(\frac{\beta\left(g_{i}\right)}{g_{i}} F_{\mu \nu}^{a} F^{a \mu \nu}+\gamma\left(m_{f}\right) \bar{\psi}_{f} \psi_{f}+\ldots\right)
$$

where $\beta\left(g_{i}\right)$ and $\gamma\left(m_{f}\right)$ are respectively the $\beta$-functions and the anomalous dimensions (here $i=$ $1,2,3$ correspond to the $S U(3), S U(2), U(1)$ coupling constants, with $F_{\mu \nu}^{a}$ the corresponding field strength tensor). The dominant interaction in (25) results from the gluon fields, from which we can deduce the effective interaction of the PGB with nucleons [14],

$$
\mathcal{L}_{\text {eff }} \approx-\frac{4}{\sqrt{1+6 \xi}} \frac{m_{N}}{\langle r\rangle} \sigma \bar{N} N
$$


where we have used the result [14] that

$$
\left\langle N\left|\frac{\beta\left(g_{s}\right)}{g_{s}} F_{\mu \nu}^{a} F^{a \mu \nu}\right| N\right\rangle \approx m_{N} \bar{\psi}_{N} \psi_{N},
$$

and we have assumed canonical normalization for the $\sigma$ field (see Eq. (23)). Thus the interchange of a $\sigma$ field between two nucleons gives rise to a static potential between two (non-relativistic) nucleons,

$$
V(r)=G_{N} \frac{32 \xi}{1+6 \xi} m_{N}^{2} \frac{\mathrm{e}^{-M_{\sigma} r}}{r}
$$

where $G_{N}$ is Newton's constant. This has to be compared with the experimentally measured gravitational potential between two nucleons. Recent torsion-balance experiments [15] showed no deviation from the standard gravitational inverse-square law, and this implies the bound,

$$
\xi \lesssim 3 \times 10^{-4} \text { for } M_{\sigma} \sim 10^{-4} \mathrm{eV} .
$$

However, for $M_{\sigma} \gtrsim 10^{-2} \mathrm{eV}$ (corresponding to a distance scale of $\sim 10^{-3} \mathrm{~cm}$ ), the limit on $\xi$ is completely relaxed. Importantly the current experiments are probing the interesting parameter range $M_{\sigma} \sim 10^{-2}-10^{-4} \mathrm{eV}, \xi \sim 1$, and thus forthcoming experiments may help in testing this theory.

\section{COSMOLOGICAL CONSTANT}

Promoting scale invariance to a good classical symmetry requires the cosmological constant to vanish classically. In other words in this type of scale-invariant theory, we would expect the cosmological constant to arise only as a radiative effect. That the cosmological constant vanishes at tree-level is an interesting feature of these theories, which optimistically speaking, might ultimately lead to an understanding of its currently small value inferred from the accelerated expansion rate of the Universe.

The lowest order contribution to the cosmological constant from the scalar sector is negative and given by [10]:

$$
E_{\text {Higgs }}=-\frac{1}{2} B\langle r\rangle^{4}
$$

Observe that this scalar vacuum energy is proportional to the one-loop contribution to the PGB mass [Eq. (15)] and is uniquely determined once the particle content of the model is specified.

An additional independent contribution to the vacuum energy-density results from the gluon condensate, which is also negative [16]:

$$
E_{Q C D}=-(9 / 32)\left\langle 0\left|\frac{\alpha_{s}}{\pi} F_{\mu \nu}^{a} F^{a \mu \nu}\right| 0\right\rangle
$$

Numerically, $\left\langle 0\left|\left(\alpha_{s} / \pi\right) F_{\mu \nu}^{a} F^{a \mu \nu}\right| 0\right\rangle \simeq 0.012 \mathrm{GeV}^{4}$.

Note that since the contribution to the vacuum energy from the effective potential and QCD vacuum are the same sign one cannot arrange a fine-tuned cancellation between them to produce 
the observed value. One can imagine a scenario where some additional source of the cosmological constant arises to cancel these terms (perhaps from the hidden sector). A particularly interesting case arises if this exotic source is of the same order as the gluon condensate (MeV). Then the contribution from the scalar sector should vanish to order MeV and Eq. (13) implies an approximate mass relation between the new fields which dominate the one loop potential and the top quark.

\section{CONCLUSION}

We have considered the possibility that scale invariance is a classical symmetry of all fundamental interactions, including gravity. Scale invariance is broken radiatively which generates standard electroweak symmetry breaking as well as the Planck scale via the VEV of a hidden sector scalar. The hierarchy problem in such theories is eliminated, with the weak/Planck scale hierarchy emerging in the (technically natural) limit where the hidden sector decouples from the ordinary sector.

Acknowledgments: This work was supported by the Australian Research Council.

[1] The earliest work, which we aware of, where the hierarchy problem is explicitly discussed in relation to the classical scale invariance is: W. A. Bardeen, FERMILAB-CONF-95-391-T.

[2] S. R. Coleman and E. Weinberg, Phys. Rev. D 7, 1888 (1973).

[3] R. Hempfling, Phys. Lett. B 379, 153 (1996) [arXiv:hep-ph/9604278].

[4] K. A. Meissner and H. Nicolai, Phys. Lett. B 648 (2007) 312 [arXiv:hep-th/0612165].

[5] J. R. Espinosa and M. Quiros, arXiv:hep-ph/0701145.

[6] W. F. Chang, J. N. Ng and J. M. S. Wu, arXiv:hep-ph/0701254

[7] R. Foot and A. Kobakhidze, Phys. Lett. B 650, 46 (2007) [arXiv:hep-ph/0702125].

[8] T. Hambye and M. H. G. Tytgat, arXiv:0707.0633 [hep-ph].

[9] A. Zee, Phys. Rev. Lett. 42, 417 (1979).

[10] E. Gildener and S. Weinberg, Phys. Rev. D 13, 3333 (1976).

[11] R. Foot, A. Kobakhidze, K. L. McDonald and R. R. Volkas, Phys. Rev. D 76, 075014 (2007) [arXiv:0706.1829 [hep-ph]].

[12] K. A. Meissner and H. Nicolai, arXiv:0710.2840 [hep-th]. 
[13] For a review, see e.g. Y. Fuji and K. Maeda, The scalar-Tensor Gravity (Kluwer Academic Publishers, 2004).

[14] M. A. Shifman, A. I. Vainshtein, V. I. Zakharaov, Phys. Lett. B 78, 443 (1978).

[15] D. J. Kapner, T. S. Cook, E. G. Adelberger, J. H. Gundlach, B. R. Heckel, C. D. Hoyle and H. E. Swanson, Phys. Rev. Lett. 98 (2007) 021101 [arXiv:hep-ph/0611184].

[16] M. A. Shifman, A. I. Vainstein and V. I. Zakharov, Nucl. Phys. B 147, 385, 448 (1979). 